\title{
METHANE IN THE ATMOSPHERE OF THE TRANSITING HOT NEPTUNE GJ436B?
}

\author{
J.-P. Beaulied ${ }^{1,2}$, G. Tinetti ${ }^{2}$, D. M. KipPing ${ }^{2,3}$, I. Ribas ${ }^{4}$, R. J. Barber ${ }^{2}$, J. Y.-K. Cho ${ }^{5}$, I. Polichtchouk ${ }^{5}$, J. Tennyson ${ }^{2}$, \\ S. N. Yurchenko ${ }^{6}$, C. A. Griffith ${ }^{7}$, V. Batista ${ }^{1}$, I. Waldmann ${ }^{2}$, S. Miller ${ }^{2}$, S. Carey ${ }^{8}$, O. Mousis ${ }^{9}$, \\ S. J. FosSEY ${ }^{2}$, AND A. AYLWARD ${ }^{2}$ \\ ${ }^{1}$ Institut d'Astrophysique de Paris, UMR7095, CNRS, Université Paris VI, 98bis Boulevard Arago, 75014 Paris, France; beaulieu@iap.fr \\ ${ }^{2}$ Department of Physics and Astronomy, University College London, Gower Street, London WC1E 6BT, UK \\ ${ }^{3}$ Harvard Center for Astrophysics, 60 Garden Street, Cambridge, MA, USA \\ ${ }^{4}$ Institut de Ciencies de l'Espai (CSIC-IEEC), Campus UAB, 08193 Bellaterra, Spain \\ ${ }^{5}$ Astronomy Unit, Queen Mary University of London, Mile End Road, London E1 4NS, UK \\ ${ }^{6}$ Institut fur Physikalische Chemie und Elektrochemie, Technische Universitat Dresden, D-01062 Dresden, Germany \\ ${ }^{7}$ Lunar and Planetary Laboratory, University of Arizona, Tucson, AZ, USA \\ ${ }^{8}$ IPAC-Spitzer Science Center, California Institute of Technology, Pasadena, CA 91125, USA \\ ${ }^{9}$ Université de Franche-Comté, Institut UTINAM, CNRS/INSU, UMR 6213, 25030 Besançon Cedex, France \\ Received 2010 July 1; accepted 2011 February 4; published 2011 March 17
}

\begin{abstract}
We present an analysis of seven primary transit observations of the hot Neptune GJ436b at 3.6, 4.5 , and $8 \mu \mathrm{m}$ obtained with the Infrared Array Camera on the Spitzer Space Telescope. After correcting for systematic effects, we fitted the light curves using the Markov Chain Monte Carlo technique. Combining these new data with the EPOXI, Hubble Space Telescope, and ground-based $V, I, H$, and $K_{s}$ published observations, the range $0.5-10 \mu \mathrm{m}$ can be covered. Due to the low level of activity of GJ436, the effect of starspots on the combination of transits at different epochs is negligible at the accuracy of the data set. Representative climate models were calculated by using a three-dimensional, pseudospectral general circulation model with idealized thermal forcing. Simulated transit spectra of GJ436b were generated using line-by-line radiative transfer models including the opacities of the molecular species expected to be present in such a planetary atmosphere. A new, ab-initio-calculated, line list for hot ammonia has been used for the first time. The photometric data observed at multiple wavelengths can be interpreted with methane being the dominant absorption after molecular hydrogen, possibly with minor contributions from ammonia, water, and other molecules. No clear evidence of carbon monoxide and carbon dioxide is found from transit photometry. We discuss this result in the light of a recent paper where photochemical disequilibrium is hypothesized to interpret secondary transit photometric data. We show that the emission photometric data are not incompatible with the presence of abundant methane, but further spectroscopic data are desirable to confirm this scenario.
\end{abstract}

Key words: planetary systems - techniques: spectroscopic

Online-only material: color figures

\section{INTRODUCTION}

As the closest transiting planet to date, GJ436b is the smallest and coolest $(\sim 700 \mathrm{~K})$ exoplanet for which both transmission and emission flux can be measured at optical-to-IR wavelengths. This hot Neptune transits a nearby bright $\left(K_{s}=6.07\right) \mathrm{M} 2.5 \mathrm{~V}$ dwarf star at 0.029 AU with a period of $\sim 2.6438986$ days (Butler et al. 2004; Gillon et al. 2007; Demory et al. 2007). Although the planet is small with a transit depth of $\sim 0.7 \%$, its atmospheric temperature and planetary parameters (Deming et al. 2007) indicate an extended atmosphere, making it an excellent candidate for the detection of atmospheric constituents using the primary transit technique. Thus, GJ436b provides a unique opportunity of extending investigations of exoplanetary atmospheres to smaller Neptune-mass planets. Recent studies indicate the distinct nature of GJ436b's orbit and composition. The exoplanet's mass and size reveal a body that is denser and thus of a different internal structure than the Jovian-sized planets. The orbital parameters indicate a somewhat eccentric orbit; thus, the planet is probably not tidally locked, like most hot Jupiters studied so far (Nettelmann et al. 2010). The composition of GJ436b, as investigated with secondary eclipse observations of GJ436 at 3.6, 4.5, 5.8, 8, 16, and $24 \mu \mathrm{m}$ (Stevenson et al. 2010, hereafter S2010), indicates an atmosphere containing a high abundance of $\mathrm{CO}$, some water, and a low abundance of methane. This composition is far out of equilibrium chemistry, for which models at the relevant temperatures suggest that both water and methane should be present and relatively abundant in the atmosphere of this hot Neptune (Sharp \& Burrows 2007; Lodders \& Fegley 2002), possibly together with $\mathrm{NH}_{3}$, hydrocarbons, and $\mathrm{H}_{2} \mathrm{~S}$. Thus, Stevenson et al. (2010) postulate the presence of two disequilibrium mechanisms: vertical mixing to bring $\mathrm{CO}$ from deeper and warmer levels where it is abundant in equilibrium and photochemistry to destroy methane and thus explain its low abundance. Here, we present an analysis of primary transit photometry at $3.6,4.5$, and $8 \mu \mathrm{m}$, which suggests, in contrast with Stevenson et al.'s (2010) study of the secondary transit photometry data, that $\mathrm{CH}_{4}$ is the most abundant carbon molecule. In order to more fully examine the composition of GJ436b's atmosphere, we also re-analyzed the secondary transit data. We derived fluxes that confirmed those obtained by Stevenson et al. (2010). However, we obtained larger errors for the measured fluxes at 3.6 and $4.5 \mu \mathrm{m}$, which allow for a composition that is also $\mathrm{CH}_{4}$-rich, consistent with our analysis of the primary transit data. In the following sections, we discuss an analysis of new primary transit data, the re-analysis of previously published secondary transit data, and examples of radiative transfer interpretations that fit both transit data with $\mathrm{CH}_{4}$ as the dominant carbon molecule, consistent with thermochemical equilibrium solutions. 
Table 1

Best-fit Transit Depths, Ratio of Radii $p$, Mid-transit Time $T_{c}$ in BJD (UTC), Duration $T$, Orbital Semimajor Axis Divided by the Stellar Radius $a / R_{*}$, Inclination $i$, and $\Upsilon / R_{*}$ Found Using the Markov Chain Monte Carlo Fit Method Described in Section 4.1

\begin{tabular}{|c|c|c|c|c|c|c|c|}
\hline Band & $p^{2}=\left(R_{p} / R_{*}\right)^{2}$ & $p=R_{p} / R_{*}$ & $T_{c}$ (day) & $T(\mathbf{s})$ & $\boldsymbol{a} / R_{*}$ & b & $\boldsymbol{\Upsilon} \Upsilon / R_{*}\left(\mathbf{d}^{-\mathbf{1}}\right)$ \\
\hline $3.6 \mu \mathrm{m}$ & $0.712 \% \pm 0.006 \%$ & $0.08439 \pm 0.00035$ & $2454859.79494 \pm 0.00008$ & $2937 \pm 15$ & $14.21_{-0.30}^{+0.30}$ & $0.8471_{-0.073}^{+0.071}$ & $58.83 \pm 0.30$ \\
\hline $4.5 \mu \mathrm{m}$ & $0.638 \% \pm 0.018 \%$ & $0.07988 \pm 0.0012$ & $2454850.54169 \pm 0.00023$ & $2907 \pm 45$ & $15.44_{-1.10}^{+1.23}$ & $0.794_{-0.034}^{+0.043}$ & $59.44_{-0.91}^{+0.94}$ \\
\hline $8.0 \mu \mathrm{m}$ & $0.6847 \% \pm 0.012 \%$ & $0.08275_{-0.00074}^{+0.00075}$ & $2454850.54169 \pm 0.00021$ & $2858 \pm 31$ & $13.34_{-0.45}^{+0.48}$ & $0.856_{-0.012}^{+0.01}$ & $60.46_{-0.67}^{+0.68}$ \\
\hline $8.0 \mu \mathrm{m}$ & $0.675 \% \pm 0.012 \%$ & $0.08219_{-0.00071}^{+0.00071}$ & $2454856.65119 \pm 0.00015$ & $2793 \pm 29$ & $14.38_{-0.54}^{+0.59}$ & $0.839_{-0.015}^{+0.013}$ & $61.88_{-0.64}^{+0.65}$ \\
\hline \multirow[t]{2}{*}{$8.0 \mu \mathrm{m}$} & $0.715 \% \pm 0.013 \%$ & $0.08455_{-0.00075}^{+0.00075}$ & $2454864.58340 \pm 0.00016$ & $2835 \pm 31$ & $14.07_{-0.64}^{+0.64}$ & $0.841_{-0.015}^{+0.016}$ & $60.94_{-0.66}^{+0.67}$ \\
\hline & & & $8 \mu \mathbf{m}$ combined & & & & \\
\hline $8.0 \mu \mathrm{m}$ & $0.6921 \% \pm 0.0072 \%$ & $0.08319_{-0.00043}^{+0.00043}$ & & $2827 \pm 18$ & $13.84_{-0.32}^{+0.33}$ & $0.8475_{-0.008}^{+0.0074}$ & $61.11_{-0.39}^{+0.39}$ \\
\hline
\end{tabular}

\section{THE PHOTOMETRIC LIGHT CURVES}

Seven primary transits of GJ436b have been observed by Spitzer Infrared Array Camera (IRAC) as part of two Spitzer programs (40685 and 50051). Two epochs have been obtained each at $3.6 \mu \mathrm{m}$ and $4.5 \mu \mathrm{m}$, and three epochs at $8 \mu \mathrm{m}$. Unfortunately, no data were obtained at $5.8 \mu \mathrm{m}$ before the end of the cryogenic life of Spitzer. Observations have been carried out in sub-array mode, and images are delivered in cubes of 64 slices of $32 \times 32$ pixels. We median-stack all the slices within a cube, and then process the data using the method described in Section 2.2 of Beaulieu et al. (2010) for HD 209458b. Note that the data set is very similar, the target being of similar brightness in the infrared.

Spitzer's IRAC instrument has four bandpasses split into two types of detectors, which display very different instrumental systematics. The $3.6 \mu \mathrm{m}$ and $4.5 \mu \mathrm{m}$ channels are indium/ antimony detectors, which are known to exhibit ostensibly periodic flux variations. These variations are a result of a nonuniform response function within each pixel, combined with small pointing variations of the Spitzer spacecraft (see MoralesCalderon et al. 2006; Beaulieu et al. 2008). Recently, it has been proposed that these bandpasses also exhibit a time dependence, as well as pixel-phase variations (S2010).

The $5.8 \mu \mathrm{m}$ bandpass (for which no observations of primary transit of GJ436b exist) and $8.0 \mu \mathrm{m}$ bandpass are arsenic-doped silicon chips exhibiting strong temporal-ramp effects, the source of which is believed to be charge trapping (Fazio et al. 2004). The $8.0 \mu \mathrm{m}$ channel is arguably the simplest to correct of all four bandpasses.

Due to the strong dependence of the transit depth on an accurate correction for systematic effects, the full details of our corrective procedure will be described and discussed in Appendix C. We summarize a few points here.

The timescales and amplitudes of the primary transit of GJ436b and of the systematics due to pixel phase at $3.6 \mu \mathrm{m}$ and $4.5 \mu \mathrm{m}$ are very similar. As a consequence, contrary to other observations where several cycles of pixel phase are present in and out of transit, the mutual phasing of the astrophysical signal and the systematics becomes a critical issue. We carefully corrected for systematics and checked for the stability of the corrections applied to the data; we derived the final values at 3.6, 4.5, and $8 \mu \mathrm{m}$ to be used in the analysis (Table 1).

Concerning the $\mathrm{S} 2010$ reduction of secondary transit observations, we obtained similar results at 5.8 and $8 \mu \mathrm{m}$, while we find greater discrepancy with the measurements at 3.6 and $4.5 \mu \mathrm{m}$. The nature of the systematics at 3.6 and $4.5 \mu \mathrm{m}$ is similar to our primary transit observations. The $3.6 \mu \mathrm{m}$ secondary transit has a mutual phasing of the astrophysical signal and the systematics close to 0 , which is the worst case scenario. It is a similar situation to the first epoch of our primary transit at $3.6 \mu \mathrm{m}$ : in that case we had two epochs, though, so we could discard the one with such mutual phasing as unreliable. Here, there is no such possibility, so we propose adopting a larger error bar at $3.6 \mu \mathrm{m}: 0.03 \% \pm 0.02 \%$. At $4.5 \mu \mathrm{m}$, we measure a transit depth of $0.01 \% \pm 0.01 \%$ in contrast with the $3 \sigma$ upper limit of $0.01 \%$ proposed by S2010. As the interpretation of the secondary transit photometric data strongly relies on the 3.6 and $4.5 \mu \mathrm{m}$-in particular the hypothesis of photochemical disequilibrium and paucity of methane-additional measurements of secondary transits at 3.6 and $4.5 \mu \mathrm{m}$ bandpasses are critically needed.

\subsection{Stellar Activity of GJ436}

A possible source of systematic errors when combining data taken over different epochs and in different bands is the influence of magnetic activity, i.e., starspots. This was discussed in detail by Beaulieu et al. (2008) for HD189733. The magnetic activity level of GJ436 can be assessed using a number of indicators. Its $\mathrm{X}$-ray luminosity has been measured to be $L_{\mathrm{x}}=9 \times 10^{25} \mathrm{erg} \mathrm{s}^{-1}$ (Sanz-Forcada et al. 2010), which is much lower than the value of the Sun of $L_{\mathrm{x}}=2 \times 10^{27} \mathrm{erg} \mathrm{s}^{-1}$. Even accounting for the $\sim 8$ times larger emitting solar surface, the flux density from the corona of GJ436 is still $\sim 3$ times smaller than the solar value. The chromospheric $\mathrm{Ca} \mathrm{H}$ and $\mathrm{K}$ indicator also suggests a very low activity object, in agreement with its low rotation velocity (Jenkins et al. 2009) and with its kinematic properties typical of an old disk star (Browning et al. 2010). Time series data from the ground (Butler et al. 2004) and space (Ballard et al. 2010) do not find evidence for photometric variations with an amplitude above $\sim 0.5 \mathrm{mmag}$ in the visible bands, which is in overall good agreement with the rest of the activity indicators. The only hint of (modest) stellar activity comes from ground-based time series photometry presented by Demory et al. (2007), which shows scatter with a peak-to-peak amplitude of $\sim 10$ mmag possibly caused by rotational modulation. This may indicate the existence of time-dependent photometric variations, but the small number of measurements deviating from the mean in the Demory et al. study prevents a more quantitative analysis.

The effect of spot activity can be further studied by measuring variations in the transit depth at different epochs, arising from changes in the spot coverage of the strip occulted by the planet. If such an effect was important, the depth of the transit would vary over time, and this could be observed as an additional scatter in a collection of depth measurements. We used the homogeneous list of transit parameters in Coughlin et al. (2008) and calculated the standard deviation of the depth measurements. If we consider 

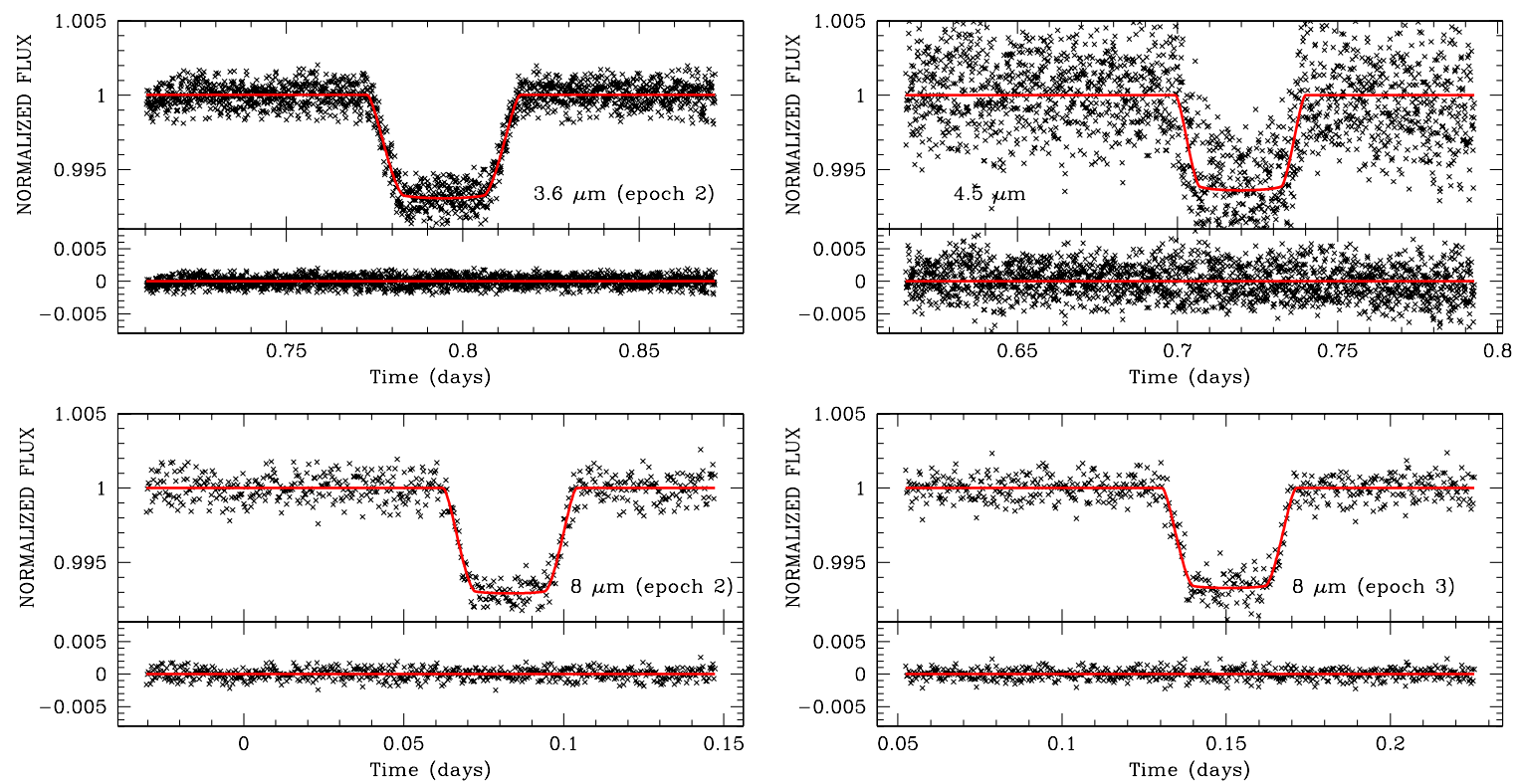

Figure 1. Final light curves, best-fit model, and residuals at $3.6 \mu \mathrm{m}, 4.5 \mu \mathrm{m}$, and the two most recent epochs at $8 \mu \mathrm{m}$. The first epoch at $8 \mu \mathrm{m}$ has been published by Deming et al. (2007), so we do not show here our re-analysis. Raw data are shown in Figure A1.

(A color version of this figure is available in the online journal.)

the six professional measurements in the $V$ band (combining all visible measurements is not adequate given the effect of limb darkening), which cover a timespan of $\sim 400$ days, the weighted depth average is $6.73 \pm 0.20 \mathrm{mmag}$ and $\chi^{2}=0.51$. Furthermore, the $I$-band measurements of Ribas et al. (2009) plus several (unpublished) additional ones totaling eight data points over a 750 day timespan (three seasons) yield a depth value of $7.02 \pm 0.17 \mathrm{mmag}$ and $\chi^{2}=1.56$

The resulting $\chi^{2}$ values, close to unity, indicate that random noise is the dominant factor and activity noise is absent or at least probably below $\sim 0.02 \%$ in the $V$ and $I$ bands. This can be translated into a conservative upper limit of $\sim 0.01 \%$ at $3.6 \mu \mathrm{m}$. Given the overall low activity level of GJ436, the effect of starspots on the combination of transits at different epochs is negligible at the quality of our data set, well below our typical transit depth measurement error.

\section{RESULTS}

\subsection{Analysis of Spitzer Primary Transits at 3.6, 4.5, and $8 \mu \mathrm{m}$}

We have five high-quality primary transit light curves with well-understood and corrected systematic effects. Note that the 2007 data at $8 \mu \mathrm{m}$ have already been published (Gillon et al. 2007; Deming et al. 2007; Southworth 2008). In this work, we processed all the data in a uniform way and present them together. First, we calculate accurate limb-darkening coefficients for each of the IRAC band following the procedure described by Beaulieu et al. (2010). With the four coefficients, Claret (2000) parameterization for limb darkening, we obtained $(0.7822,-0.8644,0.5827,-0.1557)$ for $3.6 \mu \mathrm{m},(0.6087$, $-0.5608,0.3510,-0.0918)$ for $4.5 \mu \mathrm{m}$, and $(0.5727,-0.6246$, $0.4055,-0.1026)$ for $8 \mu \mathrm{m}$.

We adopted the physical model of a transit light curve following the expression of Mandel \& Agol (2002) and orbital eccentricity following the equations of Kipping (2008). We sampled the parameter space with a Markov Chain Monte Carlo code. We adopted a fixed value of period $P=2.6438986$ days (Ballard et al. 2010). For each light curve, five parameters were fitted, namely the out-of-transit baseline, the orbital inclination $i$, the ratio between the orbital semi-major axis and the stellar radius $a / R_{*}$, the ratio of radii $k$, and the mid transit time $t_{c}$. The results are shown in Table 1 . The best-fit model and residuals for each channel are plotted in Figure 1. Note that we obtained a slightly shallower eclipse depth at $8 \mu \mathrm{m}$ than Deming et al. (2007), because of the different treatment of the ramp correction, i.e., a polynomial fit versus the Agol et al. (2009) approach, but it is compatible within the error bar. After submission of this paper, Agol et al. (2010) proposed a new approach with a double exponential function which is physically motivated with a proper asymptotic behavior also perfectly adapted for long time series obtained for phase curves. The results of the different approaches are compatible within their error bars.

\subsection{Final Values for Primary Transits to be Used for the Analysis}

We considered additional high-accuracy ground-based and space-based measurements. In particular, Cáceres et al. (2009) measured the transit depth of GJ436b in the $K_{s}$ band in 2007 May to be $0.64 \% \pm 0.03 \%$, while Alonso et al. (2008) reported $0.697 \% \pm 0.023 \%$ in the $H$ band. From Hubble Space Telescope (HST) observations in 2007 November-December, the mean transit depth between 1.35 and $1.85 \mu \mathrm{m}$ was estimated to be $0.691 \% \pm 0.012 \%$ (Pont et al. 2008). Ballard et al. (2010) combining all the data collected by the EPOXI mission obtained a transit depth of $0.663 \% \pm 0.014 \%$ over a broad band of $0.35-1.0 \mu \mathrm{m}$; this data set is not public yet. We note though that Ballard et al. (2010) performed a spline fit to the out-of-transit data before measuring the transit depth: as the data are affected by systematics with a similar timescale to the transit, this procedure may affect the derived results. We also considered the combined ground-based $V$ - and $I$-band measurements mentioned in the previous section: in $I$ the transit depth obtained was $0.702 \% \pm 0.017 \%$ and in $V$ it was $0.673 \% \pm 0.020 \%$ (Coughlin et al. 2008; Ribas et al. 2009). 

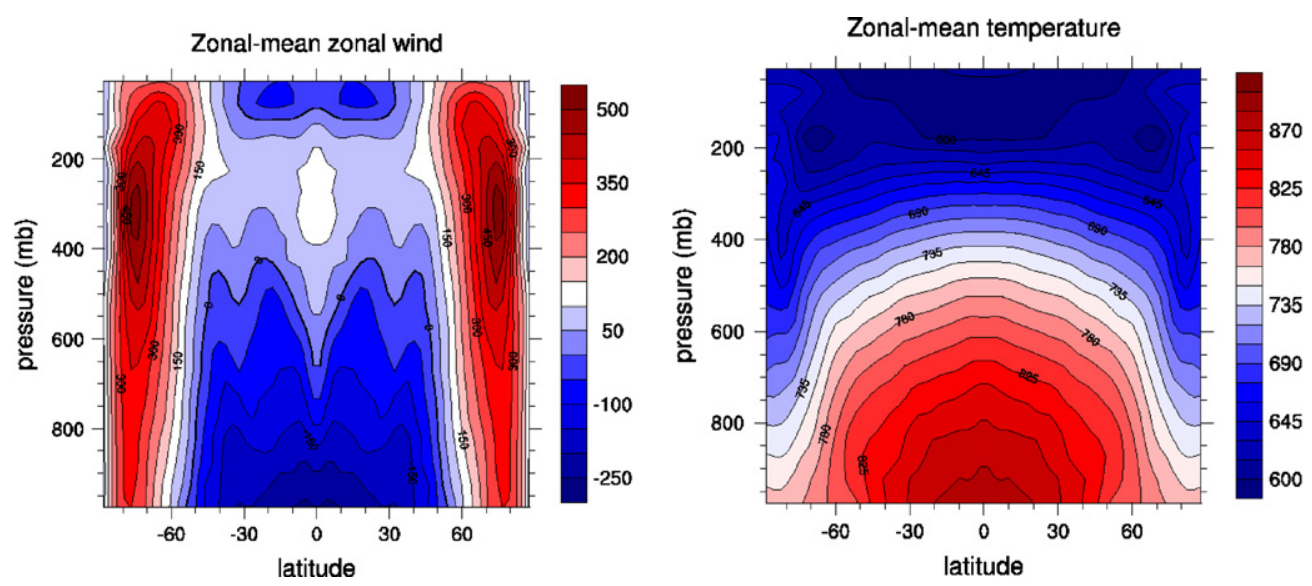

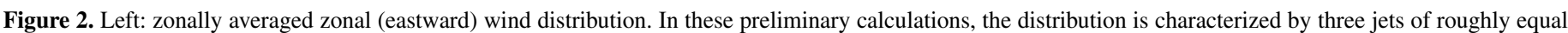

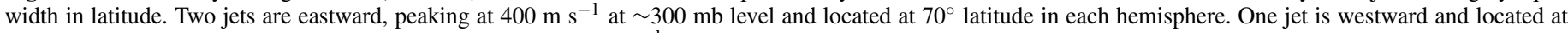

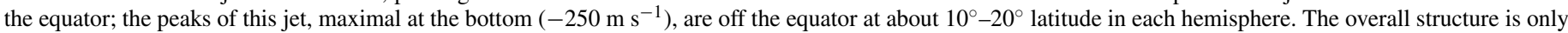

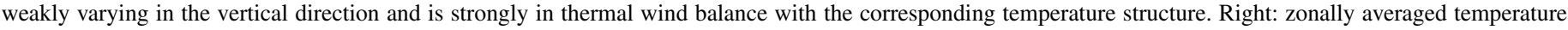

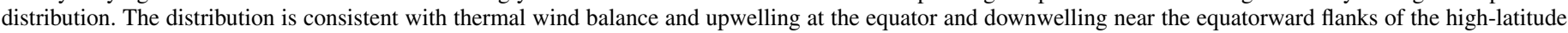

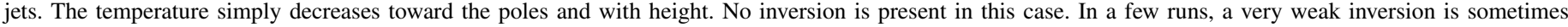
observed. However, several well-known mechanisms for producing inversions are not included in these calculations.

(A color version of this figure is available in the online journal.)

\subsection{Temperature Distribution for GJ436b}

The effective temperature of GJ436b is $\sim 649 \mathrm{~K}$, assuming that the insolation uniformly heats the entire planet (Lewis et al. 2010). However, the vertical temperature profile, as well as its variation across GJ436b's disk, is still highly unconstrained due to the paucity of spectroscopic data. Lewis et al. (2010) explored the effects of the opacity and eccentricity on the general circulation and heat distribution with a threedimensional general circulation model (GCM) that includes the radiative transfer effects due to gaseous absorption. They find that the departure of GJ436b's eccentricity from a circular orbit does not strongly affect the circulation. However, the metallicity controls the pressure of the photosphere (Spiegel et al. 2010; Lewis et al. 2010), and since the radiative time constant decreases with pressure, the temperature varies across the planet's disk (Lewis et al. 2010).

We have also explored the heat distribution in GJ436b's atmosphere. Instead of considering compositional effects, we use a Newtonian relaxation scheme applied to the heat equation, which effectively creates a photosphere at the pressure levels most equivalent to those in Lewis et al.'s solar metallicity models. As a result, we derive temperature fields that resemble those of Lewis' solar atmospheres. In our GCM calculation, the threedimensional temperature distribution on GJ436b was simulated with the global atmospheric circulation model, BOB ("Built on Beowuolf"). BOB solves the full primitive equations using a highly accurate and well-tested pseudospectral algorithm similar to that used in Thrasterson \& Cho (2010). The full details of the model can be found in Scott \& Polvani (2008). A series of simulations is performed, and a typical distribution from the series is presented in Figure 2. In the simulation shown in the figures, the characteristic thermal relaxation time is dynamically adjusted in accordance with the planet's orbit, consistent with the used Newtonian cooling approximation (Salby 1996); the equilibrium temperature distribution is barotropic (vertically uniform) and zonally symmetric (east-west symmetric), the latter in loose analogy with Venus. The horizontal resolution of the simulation corresponds to T42 in each of the 20 layers. For smooth fields, this is equivalent to at least $420 \times$
210 grid resolution in each layer, compared to that in a conventional algorithm (e.g., Canuto et al. 1988).

Figure 2 shows zonally averaged zonal (eastward) wind distribution after 100 days (planet rotations) of integration, roughly 10 thermal relaxation times. Figure 2 also shows the corresponding temperature distribution. The basic flow structure is that of two strong, high-latitude eastward jets and one weaker westward equatorial jet at the "upper tropospheric" $(\sim 300 \mathrm{mb})$ level. Note that the jets are strongly barotropic, with only one modal variation in the vertical direction. The jets are slowly strengthening and sharpening over time, which could be of dynamical significance. This will be investigated in a future work. Overall, the flow behavior is reflected by the temperature field (Figure 2), the three-dimensional structure is fairly simple and in thermal wind balance (Salby 1996): although the temperature ranges are different, the basic shape looks very similar to that of the Earth's troposphere and lower stratosphere. The temperature decreases with height at all latitudes and then hints at a tropopause at high altitudes. Dynamically generated temperature inversions are typically not present, or only very weakly present, in these set of calculations. However, the calculations do not include several well-known mechanisms for producing inversions, which may also affect the flow dynamics; these include absorbers at upper levels (Hubeny et al. 2003; Spiegel et al. 2010) and saturation of upwardly propagating waves (Watkins \& Cho 2010). Hence, any structures presented should be considered as requiring further confirmation by observations.

\subsection{Modeling the Transmission Spectra of GJ436b}

To interpret the data we simulated transmission spectra of GJ436b, which account for the effects of molecular absorption (Tinetti et al. 2007a, 2007b). Our line-by-line radiative transfer model includes opacities due to $\mathrm{H}_{2} \mathrm{O}, \mathrm{CH}_{4}, \mathrm{NH}_{3}, \mathrm{CO}$, and $\mathrm{CO}_{2}$. In our calculations here, we do not explore the range of molecular and temperature profiles that fit the data for the lack of extensive wavelength coverage and spectral resolution. We adopted temperature profiles consistent with the calculations described in Section 3.3 and with the secondary transit 


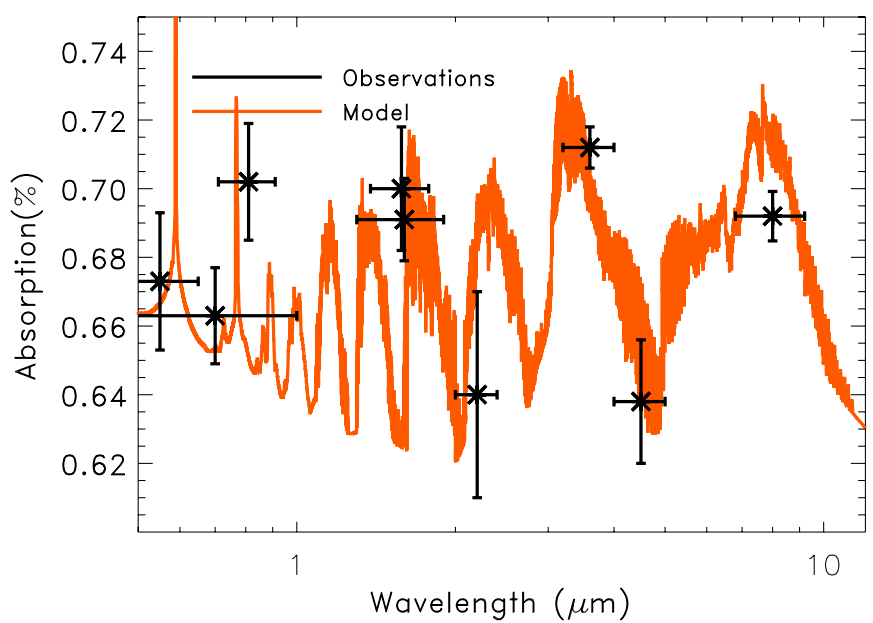

Figure 3. Simulated transmission spectrum of the transiting Hot Neptune GJ436b including the contribution of $\mathrm{CH}_{4}, \mathrm{H}_{2}$, and alkali metals in the wavelength range $0.5-9 \mu \mathrm{m}$. The simulation is a good fit to the observations with Spitzer-IRAC at 3.6, 4.5, and $8 \mu \mathrm{m}$, together with data collected by EPOXI in the range $0.5-1.0 \mu \mathrm{m}$ (Ballard et al. 2010), HST NICMOS in the range 1.2$1.8 \mu \mathrm{m}$ (Pont et al. 2009), and ground-based $H$ band (Alonso et al. 2008) and $K$ band (Cáceres et al. 2009).

(A color version of this figure is available in the online journal.)

observations by S2010. Using the BT2 line list for water (Barber et al. 2006) and the recently calculated list for ammonia (Yurchenko et al. 2010), we generated the molecular opacities at the appropriate temperatures. The complete line list for ammonia contains over two billion transitions and is the most complete and accurate source of $\mathrm{NH}_{3}$ opacity data. For the present study, transitions involving rotationally excited states up to $J=23$ were explicitly considered. Unfortunately, line lists of methane at high temperature covering the spectral range $0.5-9 \mu \mathrm{m}$ are not yet available. We combined HITRAN 2008 (Rothman et al. 2009), PNLL (Karkoschka \& Tomasko 2010), and the hightemperature measurements from Nassar \& Bernath (2003). For $\mathrm{CO}_{2}$ we used HITEMP (Rothman et al. 2010), and CDSD-1000 (Tashkun et al. 2003) for CO HITEMP was also used. The contribution of $\mathrm{H}_{2}-\mathrm{H}_{2}$ at high temperatures was taken from Borysow et al. (2001). The opacity was interpolated to the temperature of each atmospheric layer. As collision-induced absorption scales with the square of the pressure, the $\mathrm{H}_{2}-\mathrm{H}_{2}$ contribution becomes important for pressures higher than 1 bar. Given the plausible temperature range, $\mathrm{Na}$ and $\mathrm{K}$ could be present in the atmosphere of GJ436b. Their opacities were estimated from Allard et al. (2003).

Figure 3 shows the primary transit data of GJ436b, compared to a model spectrum for an atmosphere that contains mainly $\mathrm{H}_{2}$ and methane, with a mixing ratio of $\sim 5 \times 10^{-4}$. In the visible region, our simulated spectrum is dominated by Rayleigh scattering and emission due to alkali metals. While we do not explore the range of solutions allowed by the degeneracies in radius, temperature, and composition, in Figure 4, we can appreciate the contributions of other molecules. While water vapor and ammonia could potentially, but not crucially, be present, the spectral features of $\mathrm{CO}$ and $\mathrm{CO}_{2}$ display spectral patterns that oppose the transmission data, suggesting very limited, if any, abundances.

We show in Figure 5 the secondary eclipse measurements published by S2010, along with one of our spectral models at 3.6 and $4.5 \mu \mathrm{m}(0.03 \% \pm 0.02 \%$ and $0.01 \% \pm 0.01 \%$, respectively, see Appendix $\mathrm{C}$ for details). The interpretation of these data leads to a broad range of highly degenerate solutions for the molecular composition and $T-P$ profile. While a full study of the solution space is beyond the scope of this paper, we find that a combination of molecular hydrogen and methane (as suggested by transmission data) with a $T-P$ profile containing an inversion at $10^{-2}$ to $10^{-3}$ bar fit the data quite well. Such a methane-rich atmosphere is excluded by S2010 analysis of the same data, which heavily relies on the errors derived for two measurements at 3.6 and $4.5 \mu \mathrm{m}$. Their errors bars prohibit the methane-rich atmospheres, which are allowed in our study, simply because we derive higher error bars than S2010. We find that both primary and secondary transit measurements are consistent with a $\mathrm{CH}_{4}$-rich atmosphere, in agreement with thermochemical equilibrium models of exoplanet. Thus, the disequilibria processes proposed by S2010 and Madhusudhan \& Seager (2010) are not necessary to explain the data. However, there is still very little known about the thermal and compositional profiles that characterize GJ436b. Additional spectroscopic data are needed to break the degeneracy in composition and thermal profiles and distributions so that we can begin to understand the chemistry and dynamics of this Neptunian-sized planet.

Shabram et al. (2010) compare and discuss the transit spectra for GJ436b presented here using chemical species and abundances adapted from Zahnle et al. (2009a) and Stevenson et al. (2010). In this paper and in a previous one (Fortney et al. 2010),
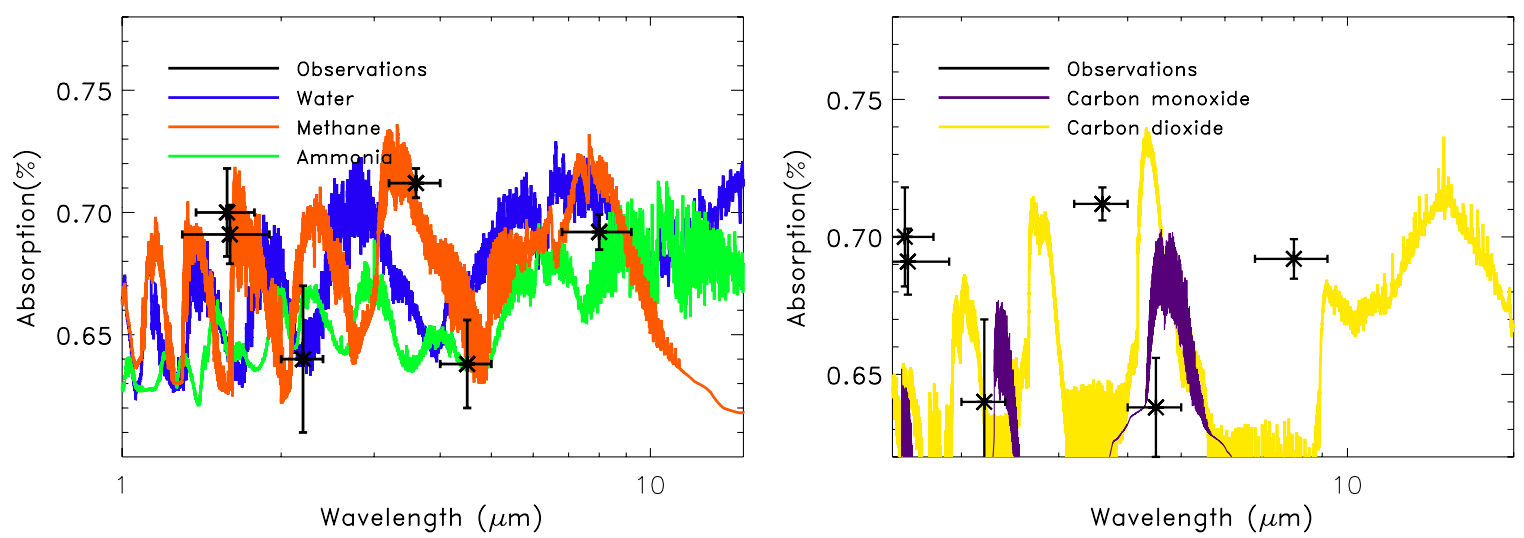

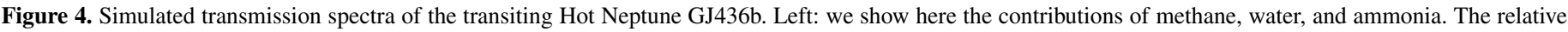

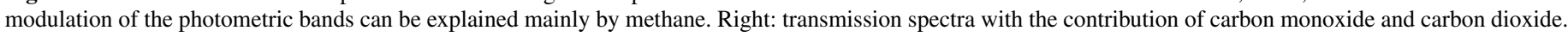
Compared to the observations, the signatures of these molecules show inconsistent behavior.

(A color version of this figure is available in the online journal.) 


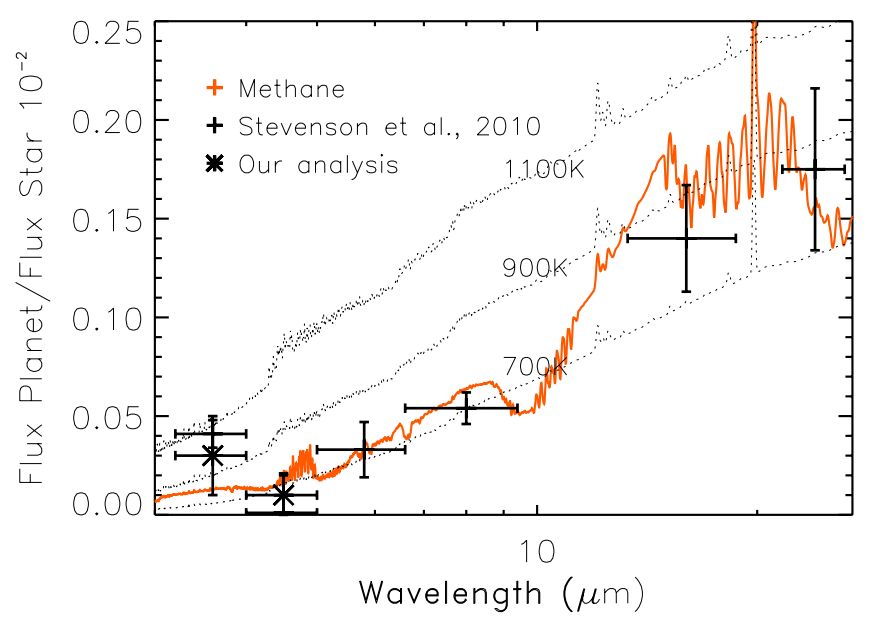

Figure 5. Secondary transit observations analyzed by S2010. Our analysis suggests larger error bars at 3.6 and $4.5 \mu \mathrm{m}$ (starred points). In color, a simulated emission spectrum with methane being predominant and a $T-P$ profile with an inversion at $\sim 10^{-2}$ bar. This solution is consistent with the interpretation of the primary transit data. Dashed lines represent blackbody curves divided by stellar model synthetic spectra.

(A color version of this figure is available in the online journal.)

they cast doubts on the validity of Tinetti's transmission models used to fit transit data for other hot-Jupiters (e.g., Tinetti et al. 2007a, 2007b; Swain et al. 2008; Beaulieu et al. 2010) on the basis that they cannot reproduce the same results with their model. While the cause of the disagreement cannot be identified without a complete knowledge of all the parameters used as input to their model (e.g., gravity field, radius at the 1 bar level etc.); we note here that Tinetti and Griffith obtain overlapping results when running their radiative transfer models in parallel, and are in agreement with the results of Madhusudhan \& Seager (2009) (see, e.g., Figure 6 of Madhusudhan \& Seager). Tinetti's radiative transfer model uses the equations and observational geometry described in Seager \& Sasselov (2000) and Brown (2001).

Shabram et al. (2010) used an analytical relation between absorption cross section and transit radius proposed by Lecavelier des Etangs et al. (2008) to validate their model. This analytical expression hypothesizes opacity cross sections that vary as a power law: while this is correct in the case of a pure Rayleigh scattering atmosphere, the relation is unphysical when we consider molecular absorption, such as in the case of water vapor, as explained in classical radiative transfer books (e.g., Goody \& Yung 1961; Liou 2002).

\section{CONCLUSIONS}

We have presented an analysis of seven primary transit observations of the hot Neptune GJ436b obtained with the SpitzerIRAC camera. The final transit depth measurements obtained are $0.712 \% \pm 0.006 \%, 0.638 \% \pm 0.018 \%$, and $0.692 \% \pm 0.007 \%$, respectively, at $3.6,4.5$, and $8 \mu \mathrm{m}$. These new data taken together with the EPOXI, $H S T$, and ground-based $V, I, H$, and $K_{s}$ observations strongly suggest that methane is the dominant absorption in GJ436b's atmosphere. We point out that secondary eclipse data, which samples the dayside atmosphere, are consistent with both methane abundant atmospheres as well as methane depleted atmosphere, proposed by Stevenson et al. if the error bars are allowed to be larger at 3.6 and $4.5 \mu \mathrm{m}$ as proposed here.

In conclusion, transmission photometry of GJ436b indicates methane as the most carbon abundant species in the atmosphere at the terminator, while the molecular form of carbon on the day side is unclear; both methane-rich and carbon monoxide-rich atmospheres fit the data. Additional spectroscopic measurements are needed.

G.T. is supported by a Royal Society University Research Fellowship, D.M.K. and I.W. by STFC, R.J.B. by the Leverhulme Trust, J.Y.-K.C. is supported by the STFC PP/ E001858/1 grant. I.R. acknowledges support from the Spanish Ministerio de Ciencia e Innovación via grant AYA2009-06934. We acknowledge the support by ANR-06-BLAN-0416 and the "Programme Origine des Planètes et de la Vie." This work is based on observations made with the Spitzer Space Telescope which is operated by the Jet Propulsion Laboratory, California Institute of Technology under a contract with NASA.

\section{APPENDIX A}

\section{PIXEL PHASE CORRECTIONS FOR 3.6 $\mu \mathrm{m}$ AND $4.5 \mu \mathrm{m}$}

The raw data at $3.6 \mu \mathrm{m}$ and $4.5 \mu \mathrm{m}$ are shown in Figure A1.

\section{A.1. Four Families of Pixel-phase Corrections}

Pixel-phase variations can be corrected for by correlating the observed flux to the pixel position. Regardless of what coordinate system one wishes to adopt, two parameters are required to describe the position of the centroid within the pixel. In addition, there is a possibility of a temporal dependence, both in the absolute flux and/or the intrapixel response function itself. This therefore extends the number of parameters required to fully describe any correlations to three. In this work, we consider four families of pixel-phase correction:

1. CN. Cartesian coordinate system for spatial parameters, without time components: $x, y$.

2. CT. Cartesian coordinate system for spatial parameters, with time components: $x, y, t$.

3. PN. Polar coordinate system for spatial parameters, without time components: $r, \theta$.

4. PT. Polar coordinate system for spatial parameters, with time components: $r, \theta, t$.

\section{A.2. Model Exploration}

For any given family, there is a wide range of possible combinations of the parameters to fit for. In this work, we will consider symmetric polynomial expansions of the dependent parameters. For each family, we have tried linear $(n=1)$, quadratic $(n=2)$, cubic $(n=3)$, and quartic $(n=4)$ symmetric polynomial expansions for the form of the pixel-phase model.

For the non-temporal families, the number of degrees of freedom in the fit will follow the triangular number set $(3,6,10,15, \ldots)$. In contrast, the temporal families will follow the tetrahedral number set $(4,10,20,35, \ldots)$.

Below, we provide the explicit forms of all of these models. For the $\mathrm{CN}$ family (Cartesian non-temporal)

$$
\begin{aligned}
n= & 1 a_{1}+a_{2} x+a_{3} y \\
n= & 2 a_{1}+a_{2} x+a_{3} y+a_{4} x^{2}+a_{5} y^{2}+a_{6} x y \\
n= & 3 a_{1}+a_{2} x+a_{3} y+a_{4} x^{2}+a_{5} x y+a_{6} y^{2}+a_{7} x^{3}+a_{8} x^{2} y \\
& +a_{9} x y^{2}+a_{10} y^{3} \\
n= & 4 a_{1}+a_{2} x+a_{3} y+a_{4} x^{2}+a_{5} x y+a_{6} y^{2}+a_{7} x^{3}+a_{8} x^{2} y \\
& +a_{9} x y^{2}+a_{10} y^{3}+a_{11} x^{4}+a_{12} x^{3} y+a_{13} x^{2} y^{2} \\
& +a_{14} x y^{3}+a_{15} y^{4} .
\end{aligned}
$$



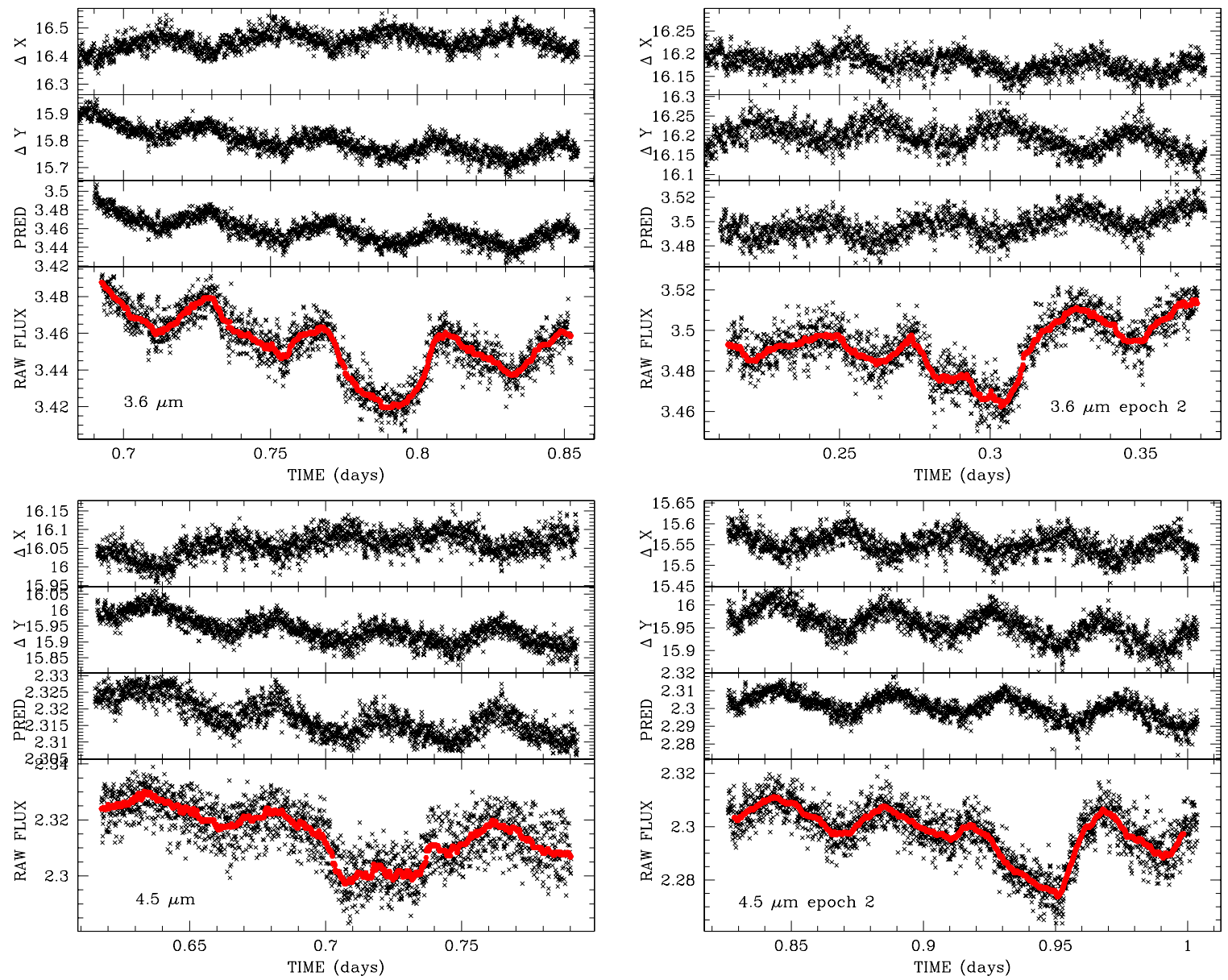

Figure A1. Raw photometric data for 3.6 and $4.5 \mu \mathrm{m}$ obtained with IRAC. Each sub-panel has the same structure showing from top to bottom: the variation of the centroid position in $X$, in $Y$, and lastly the predicted baseline flux using pixel-phase correction. The lowest panel of each plot is the primary transit and overplotted the 50-point median-stack smoothing. They provide a synoptic view of the systematic trends present in IRAC primary transit data.

(A color version of this figure is available in the online journal.)

For the CT family (Cartesian temporal), we used

$$
\begin{aligned}
n= & 1 b_{1}+b_{2} x+b_{3} y+b_{4} t \\
n= & 2 b_{1}+b_{2} x+b_{3} y+b_{4} t+b_{5} x^{2}+b_{6} x y+b_{7} x t \\
& +b_{8} y^{2}+b_{9} y t+b_{10} t^{2} \\
n= & 3 b_{1}+b_{2} x+b_{3} y+b_{4} t+b_{5} x^{2}+b_{6} x y+b_{7} x t \\
& +b_{8} y^{2}+b_{9} y t+b_{10} t^{2}+b_{11} x^{3}+b_{12} x^{2} y \\
& +b_{13} x^{2} t+b_{14} x y^{2}+b_{15} x y t+b_{16} x t^{2} \\
& +b_{17} y^{3}+b_{18} y^{2} t+b_{19} y t^{2}+b_{20} t^{3} \\
n= & 4 b_{1}+b_{2} x+b_{3} y+b_{4} t+b_{5} x^{2}+b_{6} x y+b_{7} x t \\
& +b_{8} y^{2}+b_{9} y t+b_{10} t^{2}+b_{11} x^{3}+b_{12} x^{2} y \\
& +b_{13} x^{2} t+b_{14} x y^{2}+b_{15} x y t+b_{16} x t^{2} \\
& +b_{17} y^{3}+b_{18} y^{2} t+b_{19} y t^{2}+b_{20} t^{3}+b_{21} x^{4} \\
& +b_{22} x^{3} y+b_{23} x^{3} t+b_{24} x^{2} y^{2}+b_{25} x^{2} y t+b_{26} x^{2} t^{2} \\
& +b_{27} x y^{3}+b_{28} x y^{2} t+b_{29} x y t^{2}+b_{30} x t^{3}+b_{31} y^{4} \\
& +b_{32} y^{3} t+b_{33} y^{2} t^{2}+b_{34} y t^{3}+b_{35} t^{4} .
\end{aligned}
$$

For the PN family, we have

$$
\begin{aligned}
n= & 1 c_{1}+c_{2} r+c_{3} \theta \\
n= & 2 c_{1}+c_{2} r+c_{3} \theta+c_{4} r^{2}+c_{5} \theta^{2}+c_{6} r \theta \\
n= & 3 c_{1}+c_{2} r+c_{3} \theta+c_{4} r^{2}+c_{5} r \theta+c_{6} \theta^{2} \\
& +c_{7} r^{3}+c_{8} r^{2} \theta+c_{9} r \theta^{2}+c_{10} \theta^{3}
\end{aligned}
$$

$$
\begin{aligned}
n= & 4 c_{1}+c_{2} r+c_{3} \theta+c_{4} r^{2}+c_{5} r \theta+c_{6} \theta^{2} \\
& +c_{7} r^{3}+c_{8} r^{2} \theta+c_{9} r \theta^{2}+c_{10} \theta^{3}+c_{11} r^{4} \\
& +c_{12} r^{3} \theta+c_{13} r^{2} \theta^{2}+c_{14} r \theta^{3}+c_{15} \theta^{4} .
\end{aligned}
$$

For the PT family (Polar Temporal), we used

$$
\begin{aligned}
n= & 1 d_{1}+d_{2} r+d_{3} \theta+d_{4} t \\
n= & 2 d_{1}+d_{2} r+d_{3} \theta+d_{4} t+d_{5} r^{2}+d_{6} r \theta+d_{7} r t \\
& +d_{8} \theta^{2}+d_{9} \theta t+d_{10} t^{2} \\
n= & 3 d_{1}+d_{2} r+d_{3} \theta+d_{4} t+d_{5} r^{2}+d_{6} r \theta+d_{7} r t \\
& +d_{8} \theta^{2}+d_{9} \theta t+d_{10} t^{2}+d_{11} r^{3}+d_{12} r^{2} \theta \\
& +d_{13} r^{2} t+d_{14} r \theta^{2}+d_{15} r \theta t+d_{16} r t^{2} \\
& +d_{17} \theta^{3}+d_{18} \theta^{2} t+d_{19} \theta t^{2}+d_{20} t^{3} \\
n= & 4 d_{1}+d_{2} r+d_{3} \theta+d_{4} t+d_{5} r^{2}+d_{6} r \theta+d_{7} r t \\
& +d_{8} \theta^{2}+d_{9} \theta t+d_{10} t^{2}+d_{11} r^{3}+d_{12} r^{2} \theta \\
& +d_{13} r^{2} t+d_{14} r \theta^{2}+d_{15} r \theta t+d_{16} r t^{2} \\
& +d_{17} \theta^{3}+d_{18} \theta^{2} t+d_{19} \theta t^{2}+d_{20} t^{3} \\
& +d_{21} r^{4}+d_{22} r^{3} \theta+d_{23} r^{3} t+d_{24} r^{2} \theta^{2} \\
& +d_{25} r^{2} \theta t+d_{26} r^{2} t^{2}+d_{27} r \theta^{3}+d_{28} r \theta^{2} t \\
& +d_{29} r \theta t^{2}+d_{30} r t^{3}+d_{31} \theta^{4}+d_{32} \theta^{3} t \\
& +d_{33} \theta^{2} t^{2}+d_{34} \theta t^{3}+d_{35} t^{4} .
\end{aligned}
$$


Table A1

BIC Values of All the Models We Tried to Correct the Pixel-phase Effect

\begin{tabular}{|c|c|c|c|c|}
\hline Order, $n$ & $\mathrm{CN} \mathrm{BIC}$ & CT BIC & PN BIC & PT BIC \\
\hline \multicolumn{5}{|c|}{ Channel 1} \\
\hline 1 & 1300.171 & 1304.982 & 1310.678 & 1315.350 \\
\hline 2 & 1276.464 & 1284.769 & 1278.686 & 1286.245 \\
\hline 3 & 1275.677 & 1317.442 & 1272.918 & 1314.945 \\
\hline 4 & 1293.197 & 1394.872 & 1290.468 & 1390.206 \\
\hline \multicolumn{5}{|c|}{ Channel 2} \\
\hline 1 & 1444.680 & 1429.981 & 1444.380 & 1429.626 \\
\hline 2 & 1445.941 & 1446.977 & 1446.573 & 1447.901 \\
\hline 3 & 1450.939 & 1480.064 & 1450.999 & 1479.110 \\
\hline 4 & 1475.250 & 1577.162 & 1476.360 & 1577.638 \\
\hline \multicolumn{5}{|c|}{ Channel 5} \\
\hline 1 & 1264.150 & 1196.149 & 1308.062 & 1245.248 \\
\hline 2 & 1266.709 & 1206.635 & 1268.543 & 1208.899 \\
\hline 3 & 1259.664 & 1246.510 & 1260.148 & 1249.250 \\
\hline 4 & 1274.027 & 1336.543 & 1273.779 & 1336.462 \\
\hline \multicolumn{5}{|c|}{ Channel 8} \\
\hline 1 & 1552.284 & 1556.485 & 1526.502 & 1530.995 \\
\hline 2 & 1504.001 & 1484.829 & 1506.415 & 1492.758 \\
\hline 3 & 1526.905 & 1555.750 & 1526.351 & 1551.959 \\
\hline 4 & 1560.801 & 1652.530 & 1560.612 & 1652.909 \\
\hline
\end{tabular}

Notes. For each channel, four families of corrective procedure were attempted (CN, CT, PN, and PT, where $\mathrm{C}=$ Cartesian, $\mathrm{P}=$ Polar, $\mathrm{N}=$ non-temporal, and $\mathrm{T}=$ temporal $)$ with four orders of complexity each $(n=1,2,3,4)$. The lowest BIC for each family is highlighted in bold.

\section{A.3. Model Selection}

As we increase the number of degrees of freedom, $k$, any fit we perform will naturally produce a lower merit function. The question therefore is at which point do we stop adding degrees of freedom. S2010 proposed using the Bayesian Information Criterion (BIC) to make this determination. BIC has the advantage of penalizing models for being overly complex and thus will not decrease ad infinitum as $n$ increases. The optimum model is given by that which yields the lowest BIC. Defining $x_{i}$ as the residual of a fit, $\sigma_{i}$ as the measurement uncertainty, and $N$ as the number of data points, the expression for BIC is given by

$$
\mathrm{BIC}=\sum_{i=1}^{N}\left(x_{i} / \sigma_{i}\right)^{2}+k \ln N .
$$

In Table A1, we provide the full list of all models tried to correct the pixel-phase effects for the four affected time series.

\section{A.4. Final Model Values}

For each time series, we show the best-fit model parameters in Table A2. For all dimensions (i.e., $x, y, r, \theta$, and $t$ ), the median of the array is subtracted first before fitting. This step serves to reduce interparameter correlations. For example, for $t$, which is in BJD, the BJD value is much larger than the duration of the measurement and thus fitting a function $\alpha+\beta t$ would give rise to a very large correlation between $\alpha$ and $\beta$ and thus very large errors. A better approach is to move the pivot to the median of the time stamps which serves as a far improved pivot point. The same is true for the other parameters as well.
Table A2

Best-fitted Model Parameters for Each Time Series, Where We Only Present the Parameters of the Final Favored Model (Determined Using the Bayesian Information Criterion)

\begin{tabular}{|c|c|c|}
\hline Parameter & Best Fit & Standard Error \\
\hline \multicolumn{3}{|c|}{ Channel 1} \\
\hline$c_{1}$ & 34590.6 & 5.8 \\
\hline$c_{2}$ & 1670 & 290 \\
\hline$c_{3}$ & -32.2 & 2.5 \\
\hline$c_{4}$ & 11400 & 4600 \\
\hline$c_{5}$ & -396 & 78 \\
\hline$c_{6}$ & -0.64 & 0.76 \\
\hline$c_{7}$ & -41000 & 21000 \\
\hline$c_{8}$ & 0 & 630 \\
\hline$c_{9}$ & 545 & 13 \\
\hline$c_{10}$ & 5.79 & 0.25 \\
\hline \multicolumn{3}{|c|}{ Channel 2} \\
\hline$d_{1}$ & 23199.7 & 3.4 \\
\hline$d_{2}$ & 30 & 61 \\
\hline$d_{3}$ & -13.5 & 1.0 \\
\hline$d_{4}$ & -760 & 31 \\
\hline \multicolumn{3}{|c|}{ Channel 5} \\
\hline$b_{1}$ & 34973.24 & 0.84 \\
\hline$b_{2}$ & -757 & 40 \\
\hline$b_{3}$ & -2963 & 28 \\
\hline$b_{4}$ & 159 & 18 \\
\hline \multicolumn{3}{|c|}{ Channel 8} \\
\hline$b_{1}$ & 23003.3 & 1.9 \\
\hline$b_{2}$ & -368 & 49 \\
\hline$b_{3}$ & 1812 & 49 \\
\hline$b_{4}$ & -112 & 39 \\
\hline$b_{5}$ & 4700 & 1300 \\
\hline$b_{6}$ & 4400 & 2000 \\
\hline$b_{7}$ & -800 & 1200 \\
\hline$b_{8}$ & -700 & 1200 \\
\hline$b_{9}$ & 2900 & 1300 \\
\hline$b_{10}$ & 3420 & 600 \\
\hline
\end{tabular}

Notes. Best-fit parameters computed using a Levenberg-Marquardt algorithm. All errors quoted to two significant figures and corresponding best-fit values to the equivalent number of decimal places.

\section{A.5. Fitting Procedure}

The final point we wish to address is how these fits should be performed. There are currently two schools of thought. The first is to exclude the data where the astrophysical signal is expected and use the baseline data as a calibration tool (e.g., Knutson et al. 2007; Beaulieu et al. 2010). In this approach, we simply perform a least-squares fit of a given model to the flux counts.

The second approach is to fit for both the astrophysical signal and the systematic model simultaneously. An obvious advantage of this approach is that correlations between the transit depth and the selected free parameters can be investigated. However, there are two concerns with this approach. The first one occurs when one is faced with an astrophysical signal of the same phase and timescale as the systematics. For transiting planets like HD 209458b, HD 189733b, or HD 80606, we have several cycles of the pixel-phase effect within the transit. This, unfortunately, is not the case for transiting planets such as CoRoT-7b, or even worse GJ1214b and GJ436b, where the transit duration is similar to the pixel-phase timescale.

The second critical problem is modeling the astrophysical signal. If one assumes a simple transit or eclipse then there 

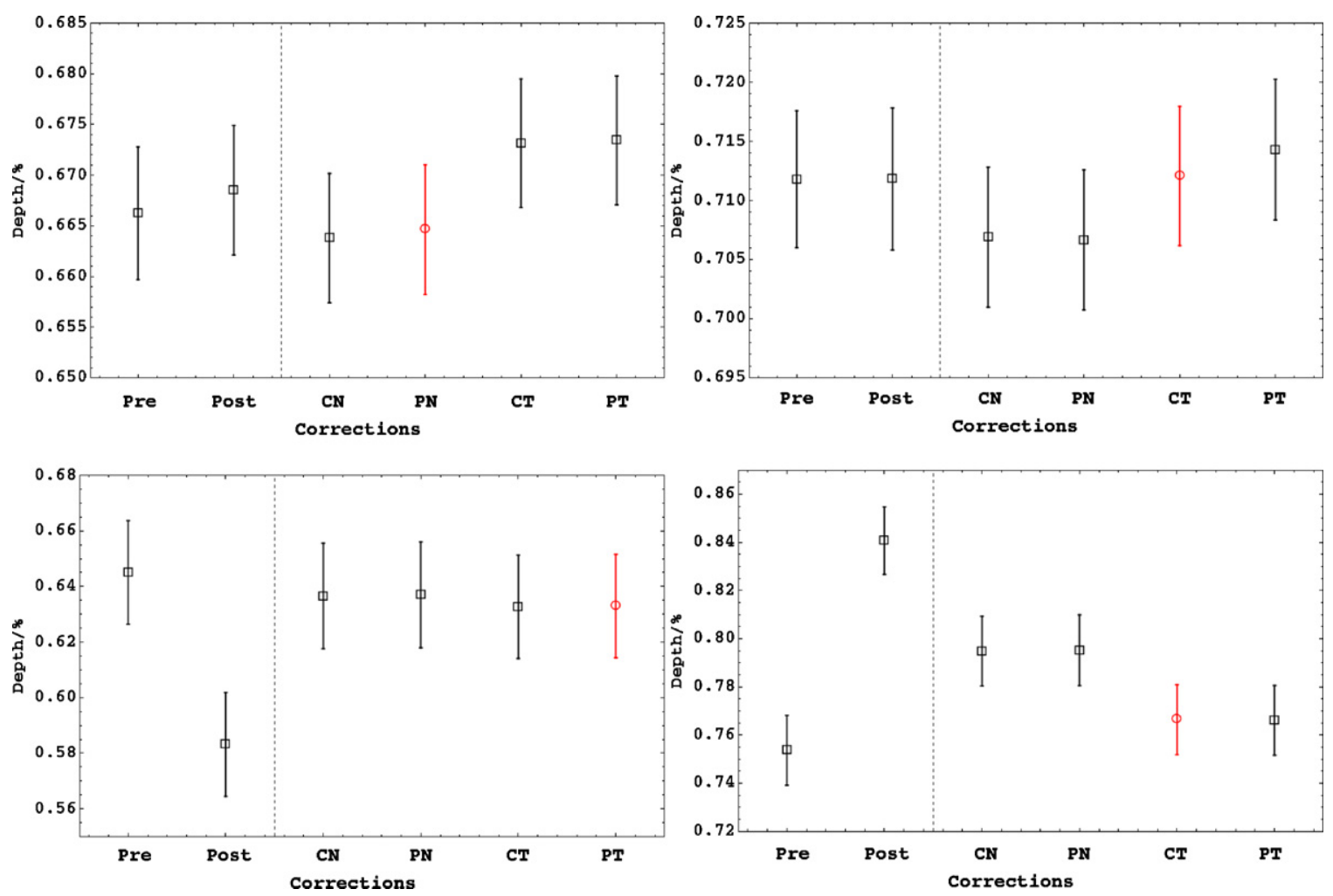

Figure A2. Measured transit depths at $3.6 \mu \mathrm{m}$ epoch 1 and 2 (left and right upper panels) and at $4.5 \mu \mathrm{m}$ (left and right lower panels), respectively, using the different families of corrections mentioned in the text. For each family, we only show the polynomial order which yielded the lowest BIC. The family which yielded the overall lowest BIC is plotted with a circle. "Pre" and "Post" are corrections using this same corrective function, but using only the pre-transit/post-transit data, respectively.

(A color version of this figure is available in the online journal.)

are no concerns. Unfortunately, a simple transit model may be insufficient at the level of precision obtained by Spitzer. Particularly when observing M dwarfs, one cannot exclude the possibility of starspots affecting the transit signal. A starspot crossing would induce a bump in the transit shape which would bear a remarkable resemblance to half of a pixel-phase period. If such a bump existed and a simultaneous fit was performed, the systematic correction would try to model the bump as part of the pixel-phase response and thus lead to essentially an erroneous correction.

Other effects such as an exomoon transit (Simon et al. 2009), planetary oblateness (Seager \& Hui 2002), atmospheric lensing (Sidis \& Sari 2010), and rings (Barnes \& Fortney 2004), to name a few, can all induce anomalous transit features as well. In essence, by fitting for systematics plus a transit signal across all of the data simultaneously, one has already chosen what one will discover.

This subtle point has not been previously emphasized in the exoplanet literature, but it does raise severe concerns about any results found using such methods, particularly for spotty stars. In the case of GJ436, the presence of star spots has already been reported by Demory et al. (2007) and more recently by Ballard et al. (2010) using EPOXI. We will return to the issue of GJ436's activity in the subsequent sections. In general, it is preferable to err on the side of caution: there, therefore, exists an additional strong motivation to exclude the transit signal when attempting to apply systematic corrections. It is this approach that will be adopted for the remainder of our analysis.

\section{A.6. The Unique Problems of GJ436}

Whilst BIC offers a clean statistical way of discriminating between the various models, there should be some caution in uncritically using this tool, particularly for GJ436. GJ436b is unique in that the duration of the transit is 60 minutes and the pixel-phase effect has a period between 50 and $60 \mathrm{~min}$ utes. The close proximity of these two timescales means that any single pixel-phase correction, even one which exhibits the lowest BIC, should be taken with caution. The problem is exacerbated by the fact that the transit of GJ436b is relatively shallow $(\sim 7 \mathrm{mmag})$ and actually comparable to the pixel-phase amplitude ( $\sim 5 \mathrm{mmag})$. We also note that the secondary eclipse has a very similar duration to the primary transit due to the argument of periapse being close to $\pi / 2$.

In this work, we therefore make several different corrections to ensure that our results are robust. From each family of possible pixel-phase corrections, we select the order $n$ which produces the lowest BIC, exploring up to quartic order. In the final tables, we will only quote the parameters from the absolute lowest BIC correction. However, in the different panels of Figure A2, we show the transit depths obtained using all four best corrections, for comparison. This allows the reader to assess the stability of the corrections. Additionally, in these plots, we reproduce the best BIC correction, but only using (1) the pre-transit baseline ("Pre") and/or (2) the post-transit baseline ("Post").

As is evident from Figure A2, the $3.6 \mu \mathrm{m}$ results seem very stable. The $4.5 \mu \mathrm{m}$ results exhibit some interesting differences. For the first epoch, the post-transit only correction leads to a dramatically lower transit depth compared to otherwise stable corrections. The reason for this low depth is evident when one inspects the residuals of the fit. With barely one cycle of pixel phase, the fitted correction is very poor for the pre-transit data. For $3.6 \mu \mathrm{m}$, making use of just one cycle was probably not such a significant hurdle because the signal to noise is much higher at $3.6 \mu \mathrm{m}$. 


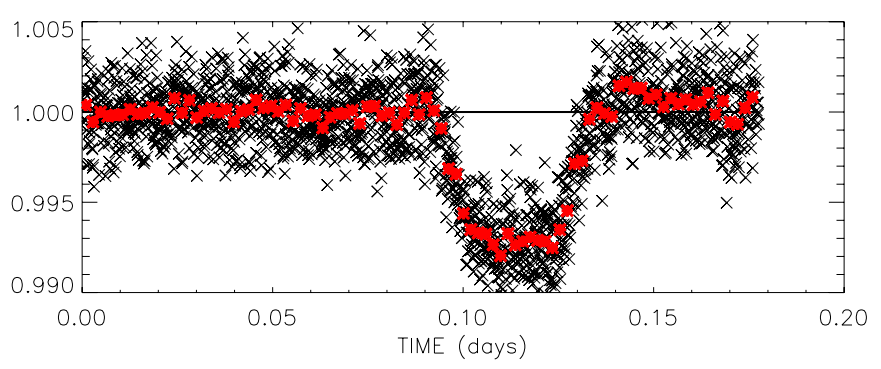

Figure A3. We present here the second epoch of $4.5 \mu \mathrm{m}$ observations where pixel phase has been corrected only using pre-transit data. Note the bump in the egress, similar to Stevenson et al. (2010) at $3.6 \mu \mathrm{m}$. We will discard this data set for the remainder of the analysis.

(A color version of this figure is available in the online journal.)

For the second epoch of $4.5 \mu \mathrm{m}$, the differences are much more severe. The pre-, post-, and combined corrections all disagree with one another. Correction using the pre-only transit applied to the full light curve reveals flat residuals except for a peculiar bump just after the egress (Figure A3). This bump could be potentially related to the star itself and a very similar feature was seen by S2010 for the same target for the secondary eclipse at $3.6 \mu \mathrm{m}$. Whilst the hypothesis of stellar induced noise is interesting, we have no way of confirming/rejecting such a hypothesis, but we note that a tiny change in the pixel-phase correction could lead to bumps of similar timescale amplitude. We therefore take the pragmatic approach of excluding this observation in our final spectral modeling. Since the first epoch of $4.5 \mu \mathrm{m}$ displays a stable correction, only this value is used in the final modeling.

\section{A.7. Transit-systematic Phasing}

The systematic trend (in this case the pixel-phase effect) and the transit duration exhibit very similar durations and amplitudes. Furthermore, the morphology of the V-shaped periodic systematic error is quite similar to that of the U-shaped transit. We are therefore in a quite undesirable scenario in terms of systematic correction. The phase difference between the periodic systematic error and the transit signal will play a crucial role in the consequences for the retrieved transit depth.

Let us define the "mutual phase," $\Delta \phi$, between these two signals. We define the flux variations caused by the pixelphase effect alone as $F_{\text {systematic }}$. This may be plotted over the uncorrected data. We inspect the fitted function around the transit and extract the time stamp of the minimum in $F_{\text {systematic }}$ both before and after the transit. The position of the mid-transit time, $t_{C}$, between these two limits, is then used to calculate the mutual phase, $\Delta \phi . \Delta \phi=0^{\circ}$, therefore indicates that the transit dips down at the exact moment the pixel-phase effect dips down. We would therefore see an increased apparent transit depth. $\Delta \phi=180^{\circ}$ means that the two signals destructively interfere to attenuate the apparent transit depth.

\section{$3.6 \mu \mathrm{m}$}

For the first and second epoch of $3.6 \mu \mathrm{m}$, we obtain $\Delta \phi=$ $0.5 \pm 5.3$ and $\Delta \phi=255.9 \pm 4.2$. Therefore, for the first epoch, the period, amplitude, and phase of the systematics effects and the astrophysical signal are almost perfectly aligned. This is the worst case scenario for attempting a correction of systematic effects. As a result of this coincidentally very unfortunate mutual phasing, we consider the transit depth obtained for the first epoch to be unreliable and most probably erroneous. It is therefore not used in our spectral modeling.
A subtle point in this issue is that the pixel-phase effect creates a periodic function with amplitude in the flux direction. There is a very slight drift in flux with respect to time, but overall it is in the flux direction. The transit depth is also in the flux direction and therefore will be most severely affected by this systematic effect. In contrast, the transit width and ingress duration, which affect parameters such as $a / R_{*}$ and impact parameter, $b$, are in the time direction. Although not completely orthogonal, these parameters will be much less severely affected by the pixel-phase effect than the transit depth. In conclusion, comparing the derived impact parameter with the known impact parameter would be a less reliable method to attempt to validate the accuracy of a pixel-phase correction.

\section{$4.5 \mu \mathbf{m}$}

For $4.5 \mu \mathrm{m}$, the phase angles for the first and second epoch are $\Delta \phi=159.4 \pm 4.5$ and $\Delta \phi=303.0 \pm 4.4$. As we have already discussed, the second epoch of $4.5 \mu \mathrm{m}$ was not used anyway because the transit depth was shown to be unstable with respect to the pixel-phase correction used. Therefore, we only need to consider the first epoch, which does not exhibit an extreme mutual phasing and thus should be reliable.

We note that the optimum strategy would be to have numerous transit observations at different values of $\Delta \phi$ which fully span the region $0^{\circ} \leqslant \Delta \phi<360^{\circ}$. By analyzing all of the transit depths at each angle, the effect of mutual phasing could potentially be removed by giving a more reliable estimate of the transit depth. However, we do not have a large number of transit measurements and so we are forced to proceed in the most reasonable way possible with the limited data presently available.

\section{APPENDIX B}

\section{RAMP CORRECTION AT $8 \mu \mathrm{m}$}

There is a variation in the response of the pixels to a long period of illumination and latent build-up effect impinges on the $8.0 \mu \mathrm{m}$ observations, called "the ramp," while their pixelphase effects are negligible. We show the three raw light curves in Figure B1. We will perform two fits to correct the data. First, following Agol et al. (2009) and Beaulieu et al. (2010) we fit the $f\left(a, b, c, d, t_{0}, t\right)=a+b\left(t-t_{0}\right)+c \log (t-$ $t_{0}$ ) function to the out-of-transit data. The second approach recently presented by Agol et al. (2010) adopts a function of the form $f\left(a_{0}, a_{1}, a_{2}, \tau_{1}, \tau_{2}, t_{0}, t\right)=a_{0}-a_{1} e^{-\left(t-t_{0}\right) / t a u_{1}}-$ $a_{2} e^{-\left(t-t_{0}\right) / \tan _{2}}$. Data corrected for systematics are shown in Figure 3. The two corrections are not distinguishable by eye and the measured transit depth agrees within the error bars. Moreover, our results are compatible with Deming et al. (2007) who reported $0.704 \% \pm 0.009 \%$ at $8 \mu \mathrm{m}$. In the final quoted values, we opt for the simpler model of Agol et al. (2009) which has just three fitted parameters. Final values for the ramp parameters are given in Table B1.

For each of three epochs, the corrected transit light curve is fitted independently using the same fitting parameter set used in Kipping \& Bakos (2011) and Kipping (2010), method A, namely $\left\{t_{c}, p^{2}, \Upsilon / R_{*}, b^{2}, O O T\right\}$. The derived parameters are listed in Table 1 . Let us define our null hypothesis to be such that the transit depths obtained for $8.0 \mu \mathrm{m}$ are consistent with the one being sampled from the same Gaussian distribution. The scatter in the depths suggests that this hypothesis is rejected at the $1.4 \sigma$ level, which is not statistically significant. We therefore conclude that there is no evidence to support a hypothesis of transit depth variation or any evidence that our systematic corrective procedure has introduced biases into any of the 


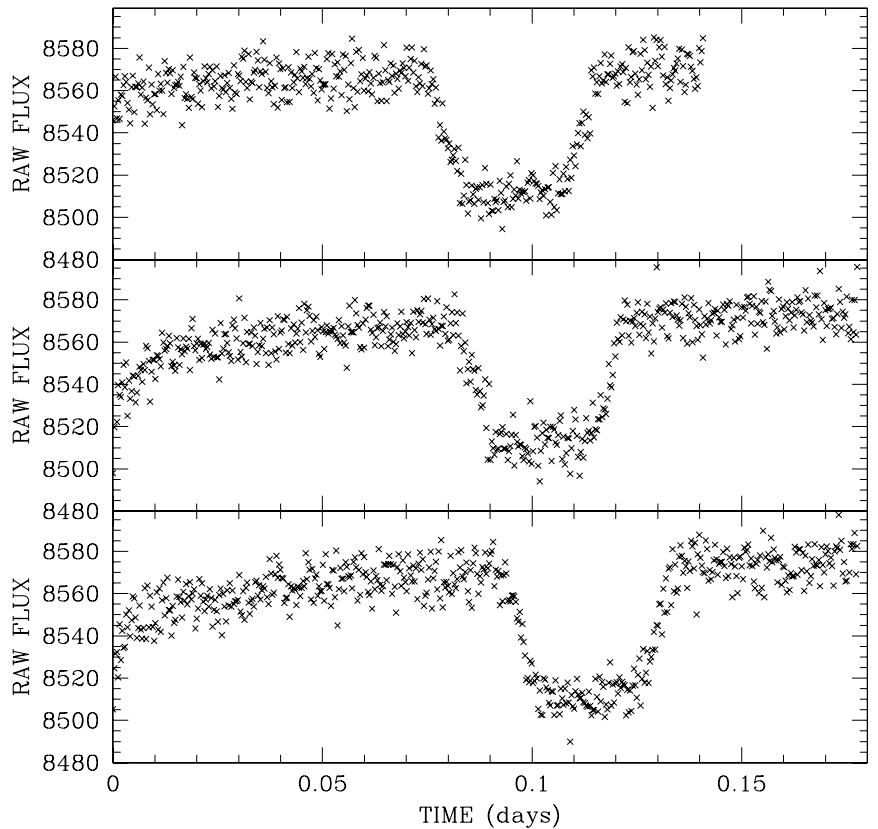

Figure B1. Raw photometric data for the three epochs at $8 \mu \mathrm{m}$ obtained with IRAC.

Table B1

Best-fitted Ramp Correction Parameters for Each $8 \mu \mathrm{m}$ Time Series

\begin{tabular}{lcc}
\hline \hline Parameter & Best Fit & Standard Error \\
\hline$a$ & Channel 4 & \\
$b$ & 8539.1 & 5.5 \\
$c$ & 0.00012 & 0.00022 \\
& 3.21 & 0.78 \\
\hline$a$ & Channel 6 & \\
$b$ & 8487.3 & 5.0 \\
$c$ & -0.00012 & 0.00015 \\
\hline & 9.33 & 0.69 \\
\hline$a$ & Channel 7 & 4.6 \\
$b$ & 8508.7 & 0.00014 \\
$c$ & 0.00002 & 0.65 \\
\hline
\end{tabular}

Notes. $t_{0}$ was selected to be $-30 \mathrm{~s}$. Best-fit parameters computed using a Levenberg-Marquardt algorithm. All errors quoted to two significant figures and corresponding best-fit values to the equivalent number of decimal places.

depths. Given this conclusion, a global fit of all three $8.0 \mu \mathrm{m}$ measurements is justified. Fitting for the period as an extra free parameter, we collectively fit all three light curves, giving the result displayed in Table B1.

\section{APPENDIX C}

\section{COMMENTS ON SPITZER SECONDARY TRANSITS OBSERVATIONS BY STEVENSON ET AL.}

We performed our own photometry and analysis of the $8 \mu \mathrm{m}$ secondary transit data used in S2010 and obtained identical results both for the transit depth and its uncertainty. We also reprocessed the data at $5.8 \mu \mathrm{m}$, correcting for systematics, and measured a secondary transit depth of $0.036 \% \pm 0.017 \%$, compatible with the $\mathrm{S} 2010$ results.

We have reexamined the $3.6 \mu \mathrm{m}$ and $4.5 \mu \mathrm{m}$ observations using similar procedures to those described here for primary

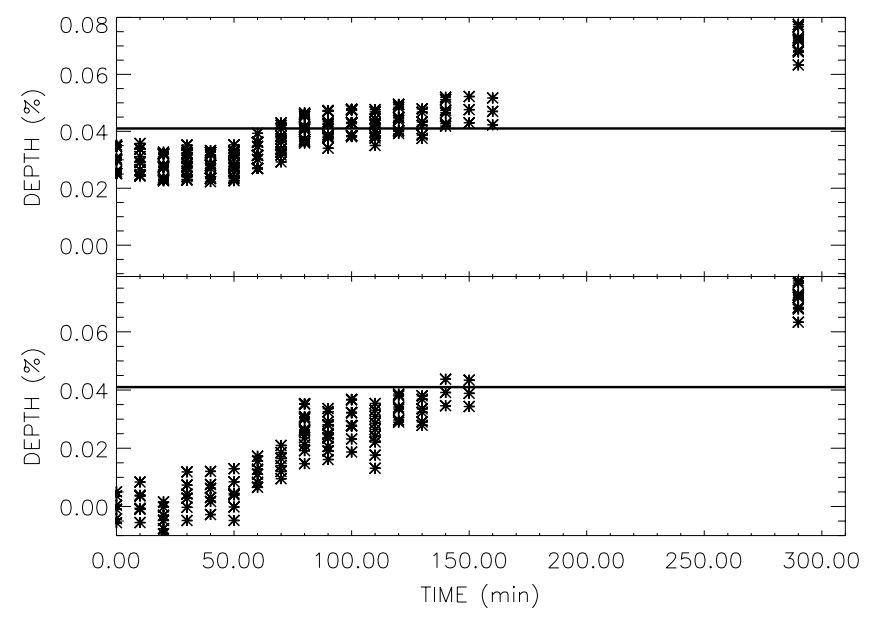

Figure C1. We present here measurements of the secondary transit depth at 3.6 $\mu \mathrm{m}$ as a function of the start time of the in-transit observations for different length of time to derive the corrective terms for systematics. See the text for detail about the procedure.

transits. For the $3.6 \mu \mathrm{m}$ data, the best correction is obtained by using quadratic terms for pixel phase and no time dependence, giving a secondary transit depth of $0.041 \% \pm 0.006 \%$. This value is identical to S2010, but the uncertainty is twice as large. However, we also note that the post-transit spike, reported in S2010 and noted as being possibly due to stellar activity, may bias the pixel-phase correction, as was also found here for $4.5 \mu \mathrm{m}$ data in primary transit. As a check, we decided to exclude the $3.6 \mu \mathrm{m}$ post-transit data and spike, the first 70 minutes of observations and recomputed the pixel-phase correction and eclipse depth: we obtain a secondary transit depth of $0.02 \% \pm 0.006 \%$. These two results are incompatible and do not incorporate an error from the systematics. The two results do not incorporate any systematic uncertainty from the pixel-phase correction, but are formally inconsistent. We then decided to explore the sensitivity to the chosen section of out-oftransit data used to estimate for the pixel-phase effect to correct the light curve. First, we considered only pre-transit data to estimate the correction. We took sections of data at 80 minutes, 100 minutes, 120 minutes, 140 minutes, and 160 minutes with different start time. We then compute the correction and fit the transit depth. We also perform similar measurement for the post-transit observations alone. Results are reported in the lower panel of Figure C1. We incorporated post-transit between 290 and 310 minutes after the first exposure and performed the same corrections and fits. It is clear that depending on the section of the data that is used to derive the correction different results are obtained, and that the transit depth measurement is completely dominated by systematic errors that are not under control. Results are shown in the upper panel of Figure $\mathrm{C} 1$. We consider that no reliable measurement could be obtained for this epoch. We also would like to comment about the post-spike seen from S2010 and also observed in our different rereduction. Rather than a photometric variation timed at the end of the secondary transit, we suggest that it could also be remaining systematics of the instrument similar to what we observed in Figure A3.

We also estimate the mutual phase between transit and pixel phase to be $\Delta \phi=5^{\circ} \pm 10^{\circ}$. This close mutual phasing and the presence of the post-transit spike would normally lead us to exclude the $3.6 \mu \mathrm{m}$ data set from further analysis; indeed, this highlights the acute need for further observations of the secondary transit of GJ436b at $3.6 \mu \mathrm{m}$ at different mutual 
phases in order to check this transit depth, especially given the high leverage exerted by this point in the S2010 analysis of the degree of methane absorption in secondary transit. We will adopt a conservative $0.03 \% \pm 0.02 \%$.

We also performed the secondary transit analysis of $4.5 \mu \mathrm{m}$ data and find a measured transit depth of $0.01 \% \pm 0.01 \%$, while $\mathrm{S} 2010$ found a $3 \sigma$ upper limit at $0.01 \%$. We conclude that the two main discrepancies between S2010 and our own analysis are at 3.6 and $4.5 \mu \mathrm{m}$, i.e., those points which carry the greatest weight in their analysis and conclusions. Measurements at 3.6 and $4.5 \mu \mathrm{m}$ should be redone with warm Spitzer. We encourage further studies on transiting exoplanets to obtain multiple epoch for transit measurements, in particular when the transit duration is of the same timescale as the pixel scale effects such as for COROT 7b, GJ1214b, and GJ436b.

\section{REFERENCES}

Agol, E., Cowan, N. B., Knutson, H. A., Deming, D., Steffen, J. H., Henry, G. W., \& Charbonneau, D. 2010, ApJ, 721, 1861

Agol, E., et al. 2009, in IAU Symp. 253, Transiting Planets, ed. F. Pont, D. Sasselov, \& M. Holman (Cambridge: Cambridge Univ. Press), 209

Allard, N. F., Allard, F., Hauschildt, P. H., Kielkopf, J. F., \& Machin, L. 2003, A\&A, 411, 473

Alonso, R., et al. 2008, A\&A, 487, L5

Ballard, S., et al. 2010, ApJ, 716, 1047

Barber, R. J., Tennyson, J., Harris, G. J., \& Tolchenov, R. N. 2006, MNRAS, 368, 1087

Barnes, J. W., \& Fortney, J. 2004, ApJ, 616, 1193

Beaulieu, J. P., Carey, S., Ribas, I., \& Tinetti, G. 2008, ApJ, 677, 1343

Beaulieu, J. P., et al. 2010, MNRAS, 409, 266

Borysow, A., Jorgensen, U. G., \& Fu, Y. 2001, J. Quant. Spectrosc. Radiat. Transfer, 68, 235

Brown, T. M. 2001, ApJ, 553, 1006

Browning, M. K., Basri, G., Marcy, G. W., West, A. A., \& Zhang, J. 2010, AJ, 139,504

Butler, R. P., et al. 2004, ApJ, 617, 580

Cáceres, C., et al. 2009, A\&A, 507, 481

Canuto, C., Hussaini, M. Y., Quarteroni, A., \& Zang, T. A. 1988, Spectral Methods in Fluid Dynamics (New York: Springer)

Claret, A. 2000, A\&A, 363, 1081

Coughlin, J. L., et al. 2008, ApJ, 689, L149

Deming, D., et al. 2007, ApJ, 667, L199

Demory, B.-O., et al. 2007, A\&A, 475, 1125

Fazio, G. G., et al. 2004, ApJS, 154, 10

Fortney, J. J., et al. 2010, ApJ, 709, 1396

Gillon, M., et al. 2007, A\&A, 471, L51

Goody, R. M., \& Yung, Y. L. 1961, Atmospheric Radiation (Oxford: Oxford Univ. Press)

Hubeny, I., Burrows, A., \& Sudarsky, D. 2003, ApJ, 594, 1011
Jenkins, J. S., et al. 2009, ApJ, 704, 975

Karkoschka, E., \& Tomasko, M. G. 2010, Icarus, 205, 674

Kipping, D. 2008, MNRAS, 389, 1383

Kipping, D. 2010, MNRAS, 408, 1758

Kipping, D., \& Bakos, G. 2011, ApJ, 730, 50

Knuston, H. A., et al. 2007, Nature, 447, 183

Lecavelier Des Etangs, A., Pont, F., Vidal-Madjar, A., \& Sing, D. 2008, A\&A, 481,83

Lewis, N. K., Showman, A. P., Fortney, J. J., Marley, M. S., Freedman, R. S., \& Lodders, K. 2010, ApJ, 720, L344

Liou, K. N. 2002, An Introduction to Atmospheric Radiation (2nd ed.; San Diego, CA: Academic)

Lodders, K., \& Fegley, B. 2002, Icarus, 155, 393

Madhusudhan, N., \& Seager, S. 2009, ApJ, 707, 24

Madhusudhan, N., \& Seager, S. 2010, ApJ, 729, 41

Mandel, K., \& Agol, E. 2002, ApJ, 580, L171

Morales-Caldern, M., et al. 2006, ApJ, 653, 1454

Nassar, R., \& Bernath, P. 2003, J. Quant. Spectrosc. Radiat. Transfer, 82, 279

Nettelmann, N., Kramm, U., Redmer, R., \& Neuhaeuser, R. 2010, A\&A, 523, A26

Pont, F., Knutson, H., Gilliland, R. L., Moutou, C., \& Charbonneau, D. 2008, MNRAS, 385, 109

Pont, F., et al. 2009, MNRAS, 393, L6

Ribas, I., Font-Ribera, A., Beaulieu, J. P., Morales, J. C., \& Garcìa-Melendo, E. 2009, in IAU Symp. 253, The Case for a Close-in Perturber to GJ436b, ed. F. Pont, D. Sasselov, \& M. Holman (Cambridge: Cambridge Univ. Press), 149

Rothman, L. S., et al. 2009, J. Quant. Spectrosc. Radiat. Transfer, 110, 533

Rothman, L. S., et al. 2010, J. Quant. Spectrosc. Radiat. Transfer, in press

Salby, M. L. 1996, Fundamental of Atmospheric Physics (San Diego, CA: Academic)

Sanz-Forcada, J., et al. 2010, A\&A, 511, L8

Scott, R. K., \& Polvani, L. M. 2008, Geophys. Res. Lett., 35, 24

Seager, S., \& Hui, L. 2002, ApJ, 574, 1004

Seager, S., \& Sasselov, D. D. 2000, ApJ, 537, 916

Shabram, M., et al. 2010, ApJ, 727, 65

Sharp, C. M., \& Burrows, A. 2007, ApJ, 168, 140

Sidis, O., \& Sari, R. 2010, ApJ, 720, 904

Simon, A. E., Szabo, Gy. M., \& Szatmary, K. 2009, Earth Moon Planets, 105, 385

Southworth, J. 2008, MNRAS, 386, 1644

Spiegel, D. S., Burrows, A., Ibgui, L., Hubeny, I., \& Milsom, J. A. 2010, ApJ, 709,149

Stevenson, K. B., et al. 2010, Nature, 464, 1161 (S2010)

Swain, M. R., Vasisht, G., \& Tinetti, G. 2008, Nature, 452, 329

Tashkun, S. A., Perevalov, V. I., Teffo, J.-L., Bykov, A. D., \& Lavrentieva, N. N. 2003, J. Quant. Spectrosc. Radiat. Transfer, 82, 165

Thrasterson, H. T., \& Cho, J. Y.-K. 2010, ApJ, 716, 144

Tinetti, G., et al. 2007a, Nature, 448, 169

Tinetti, G., et al. 2007b, ApJ, 654, L99

Watkins, C., \& Cho, J. Y.-K. 2010, ApJ, 714, 904

Yurchenko, S. N., Barber, R. J., \& Tennyson, J. 2010, MNRAS, submitted

Zahnle, K., et al. 2009, ApJ, 701, L20 